\title{
Some polynomial solutions for the non-axisymmetric Boussinesq problem
}

\author{
J.R. BARBER \\ Department of Mechanical Engineering and Applied Mechanics, University of Michigan, Ann Arbor, MI 48104, \\ U.S.A.
}

(Received July 13, 1982)

\begin{abstract}
Solutions to the Boussinesq problem for the circular punch without axial symmetry can be obtained from corresponding axisymmetric solutions by parametric differentiation. The method is applied to the problem of the tilted flat punch, originally solved by Green, and to the indentation of a cylindrical surface by a flat circular punch.
\end{abstract}

\section{Introduction}

A solution for the indentation of the semi-infinite elastic solid by a rigid circular punch of arbitrary profile was given by Green [1]. The profile of the punch is expressed as a Fourier series in polar co-ordinates centred on the axis of the punch, and use is made of a series of potential functions due to Copson [2].

A corresponding series of solutions to the non-axisymmetric problem can be obtained by differentiating axisymmetric solutions with respect to a space variable. The method is particularly straightforward where the punch profile can be expressed as a polynomial in rectangular co-ordinates.

\section{The tilted punch}

It is well known that the axisymmetric normal contact stress distribution

$$
\left.\begin{array}{rlr}
\sigma_{z z} & =-\frac{2 c_{1} E}{\pi\left(1-\nu^{2}\right)}\left(1-\frac{r^{2}}{a^{2}}\right)^{1 / 2}, & r \leqslant a \\
& =0, & r>a
\end{array}\right\}
$$

produces a parabolic depression within the contact area, defined by the displacement

$$
u_{z}=c_{1} a\left(1-\frac{r^{2}}{2 a^{2}}\right), \quad r \leqslant a,
$$

where $E, \nu$ and Young's modulus and Poisson's ratio respectively for the material of the 
semi-infinite solid, $a$ is the radius of the contact circle and $c_{1}$ is an arbitrary constant (see, for example, Timoshenko and Goodier [3], Art. 139).

If we now define a cartesian co-ordinate system on the surface of the solid by the transformation $x=r \cos \theta ; y=r \sin \theta$; it follows that the distribution

$$
\begin{aligned}
\sigma_{z z} & =a \frac{\partial}{\partial x}\left(-\frac{2 c_{1} E}{\pi\left(1-\nu^{2}\right)}\left(1-\frac{r^{2}}{a^{2}}\right)^{1 / 2}\right) \\
& =\frac{2 c_{1} E x}{\pi\left(1-\nu^{2}\right)\left(a^{2}-r^{2}\right)^{1 / 2}}, \quad r \leqslant a \\
& =0, \quad r>a
\end{aligned}
$$

will produce a depression

$$
\begin{aligned}
u_{z} & =a \frac{\partial}{\partial x}\left(c_{1} a\left(1-\frac{r^{2}}{2 a^{2}}\right)\right) \\
& =-c_{1} x, \quad r \leqslant a
\end{aligned}
$$

and corresponds to a moment

$$
M=-\frac{4 c_{1} E a^{3}}{3\left(1-\nu^{2}\right)}
$$

applied about the $y$ axis. In both the above solutions, the half-space is free of tangential tractions $\left(\sigma_{x z}=\sigma_{y z}=0\right.$ on $\left.z=0\right)$.

Equation (4) defines a plane surface, tilted through an angle $-c_{1}$ about the $y$ axis, which is the case considered by Green [1].

By superposing the solution for axisymmetric indentation by a flat circular punch

$$
\begin{array}{ll}
\sigma_{z z}=-\frac{c_{0} E}{\pi\left(1-\nu^{2}\right)}\left(1-\frac{r^{2}}{a^{2}}\right)^{-1 / 2}, & r \leqslant a, \\
u_{z}=c_{0} a, & r \leqslant a, \\
P=\frac{2 c_{0} E a^{2}}{\left(1-\nu^{2}\right)}, &
\end{array}
$$

where $P$ is the total load applied to the punch, we can obtain the contact stress distribution and the tilt produced when a rigid circular flat punch is pressed into a semi-infinite elastic solid by a force $P$ which is displaced from the axis of the punch by a distance $b$ in the $x$ direction. In this case,

$$
\begin{array}{ll}
\sigma_{z z}=\frac{-P}{2 \pi a\left(a^{2}-r^{2}\right)^{1 / 2}}\left\{1-\frac{3 b x}{a^{2}}\right\}, & r \leqslant a, \\
u_{z}=\frac{P\left(1-\nu^{2}\right)}{2 E a}\left\{1-\frac{3 b x}{2 a^{2}}\right\}, & r \leqslant a .
\end{array}
$$

Since tensile contact stresses are not normally possible, it follows from equation (9) that contact will only be maintained over the entire punch face if the indenting force is applied within a circle of radius $a / 3$. 


\section{Higher order polynomials}

A more general form of the axisymmetric solution (equations $(1,2 ; 6,7)$ ) is

$$
\sigma_{z z}=-\frac{2^{2 n} n ! n !}{(2 n) !} \frac{c_{n} E}{\pi\left(1-\nu^{2}\right)}\left(1-\frac{r^{2}}{a^{2}}\right)^{n-1 / 2}, \quad r \leqslant a
$$

which produces a displacement

$$
u_{z}=c_{n} a I_{n}, \quad r \leqslant a,
$$

where $I_{n}$ is a polynomial of degree $n$ in $\left(r^{2} / a^{2}\right)$ defined by

$$
I_{n}=\frac{1}{\pi} \int_{0}^{\pi}\left(1-\frac{r^{2}}{a^{2}} \cdot \sin ^{2} \phi\right)^{n} d \phi .
$$

This result is easily verified by integrating the point load solution, as in Timoshenko and Goodier [3], Art. 124.

The first few polynomials are

$$
\left.\begin{array}{l}
I_{0}=1, \\
I_{1}=1-\frac{r^{2}}{2 a^{2}}, \\
I_{2}=1-2\left(\frac{r^{2}}{2 a^{2}}\right)+\frac{1.3}{2.4} \frac{r^{4}}{a^{4}}, \\
I_{3}=1-3\left(\frac{r^{2}}{2 a^{2}}\right)+3\left(\frac{1.3}{2.4} \frac{r^{4}}{a^{4}}\right)-\frac{1.3 .5}{2.4 .6} \frac{r^{6}}{a^{6}}
\end{array}\right\}
$$

etc.

Other representations of these polynomial solutions are given by Gladwell, England and Shail $[4,5,6]$.

If we differentiate these solutions with respect to $x$ and/or $y$, we shall develop a corresponding series of non-axisymmetric solutions. Suppose we have a circular punch whose profile is a known polynomial in $x$ and $y$, the highest order term of which is $x^{i} y^{j}$. We first take the solution of the form $a^{i+j}\left(\partial^{i} / \partial x^{i}\right)\left(\partial^{j} / \partial y^{j}\right) I_{i+j}$ which will give such a term and which when subtracted out will leave a lower order residual polynomial. The procedure can then be repeated with the latter until a complete solution is obtained.

\section{Indentation of a cylindrical surface}

As an example, we find the pressure distribution over $r \leqslant a$ required to cause a displacement

$$
u_{z}=u-c x^{2}
$$

in the same region. This solution corresponds to the indentation of a plane surface by a punch of parabolic cross section in the $x z$ plane, but, if $c$ is small, it also gives the solution for the impression of a flat circular punch into the curved surface of a cylinder whose radius is

$$
R=\frac{1}{2 c}
$$

and is large compared with that of the punch $(a)$. 
From Eqn. (14) we have

$$
a^{2} \frac{\partial^{2} I_{2}}{\partial x^{2}}=-2+\frac{3 r^{2}}{2 a^{2}}+3 \frac{x^{2}}{a^{2}},
$$

and hence

$$
\begin{aligned}
u-c x^{2}= & -c a^{4} \frac{\partial^{2} I_{2}}{\partial x^{2}}-c a^{2} I_{1} \\
& +\frac{c a^{2}}{3} I_{0}+u I_{0} .
\end{aligned}
$$

The contact stress distribution required to produce this depression is

$$
\begin{aligned}
\sigma_{z z}= & \frac{-E}{\pi\left(1-\nu^{2}\right) a}\left\{-\frac{2.4}{1.3} \frac{c a^{4}}{3} \frac{\partial^{2}}{\partial x^{2}}\left(1-\frac{r^{2}}{a^{2}}\right)^{3 / 2}-2 c a^{2}\left(1-\frac{r^{2}}{a^{2}}\right)^{1 / 2}\right. \\
& \left.+c \frac{a^{2}}{3}\left(1-\frac{r^{2}}{a^{2}}\right)^{-1 / 2}+u\left(1-\frac{r^{2}}{a^{2}}\right)^{-1 / 2}\right\} \\
= & \frac{-E}{3 \pi\left(1-\nu^{2}\right)\left(a^{2}-r^{2}\right)^{1 / 2}}\left\{\left(3 a^{2}-8 x^{2}-2 r^{2}\right) c+3 u\right\},
\end{aligned}
$$

corresponding to a total load

$$
P=\frac{2 E a}{3\left(1-\nu^{2}\right)}\left\{3 u-c a^{2}\right\} .
$$

To prevent tensile stresses, we must have

$$
u>\frac{7 c a^{2}}{3} \text {, }
$$

i.e.,

$$
P>\frac{4 E c a^{3}}{\left(1-\nu^{2}\right)} .
$$

At lower loads, the punch will not make contact over its entire base.

\section{Internal stresses and displacements}

The same method can be used to write down the displacement within the body in terms of derivatives of the corresponding axisymmetric solutions. Since solutions to several axisymmetric problems have been studied in detail, this procedure could be more economical than treating the non-axisymmetric case from first principles. Internal stresses are readily expressed in terms of displacements. 


\section{References}

[1] A.E. Green, Proc. Cambridge Phil. Soc. 45 (1949) 251.

[2] E.T. Copson, Proc. Edinburgh Math. Soc. 8 (1947) 14.

[3] S.P. Timoshenko and J.N. Goodier, Theory of Elasticity, 3rd edn., McGraw-Hill, New York (1970).

[4] G.M.L. Gladwell and A.H. England, Q.J. Mech. Appl. Math., 30 (1977) 175.

[5] A.H. England and R. Shail, Q.J. Mech. Appl. Math. 30 (1977) 397.

[6] R. Shail, Q.J. Mech. Appl. Math. 32 (1980) 235. 